\title{
A Case of Devic's Syndrome Presenting with Tonic Spasm: Response to Levetiracetam Treatment
}

\author{
Alev Leventoğlu' ${ }^{1}$ Demet Karadağ${ }^{2}$, Zülküf Mehmet Önal ${ }^{1}$ \\ ${ }^{1}$ Department of Neurology, Faculty of Medicine, Ufuk University, Ankara, Turkey \\ ${ }^{2}$ Department of Radiology, Faculty of Medicine, Ufuk University, Ankara, Turkey
}

\begin{abstract}
Neuromyelitis optica or Devic's syndrome is a rare autoimmune disorder which is characterized by inflammatory demyelination of the optic nerves and the spinal cord. Clinically, it causes visual loss in one or both eyes, and numbness or paralysis of the arms and legs. Although tonic spasm is the most frequent movement disorder occuring in MS, it has not been definetely described clinical entity for Devic's syndrome. We hereby describe a case of Devic's syndrome with tonic spasms treated with levetiracetam as a new approach and discussed the results of the treatment. A 52-year-old woman with Devic's syndrome with the complaint of painful tonic spasms primarily affecting the abdomen was given levetiracetam therapy. Levetiracetam therapy resulted in a good response in our patient. Levetiracetam can be a new choice for the treatment of painful tonic spasm with Devic's syndrome. However, more detailed studies are necessary to investigate efficacy of levetiracetam.
\end{abstract}

Key Words: Levatiracetam, tonic spasm, devic's syndrome

\section{Introduction}

The clinical features of Devic's Syndrome (Neuromyelitis optica, Devic's disease) include temporary blindness in one or both eyes, weakness or paralysis, painful spasms, and bladder and bowel problems. Clinical manifestations of this syndrome are caused by optic neuritis and tranverse myelitis. It may follow a monophasic or relapsing course. Tonic spasm (paroxysmal dystonias, tonic seizures) is characterized by painful unilateral contractions of the upper or lower extremities $(1,2)$. Sometimes the attacks are stimulated by tactile stimulation or movement and are predated by transient sensory disturbance. Although most patients with Devic's Syndrome have a normal cerebral MRI, inflammatory demyelinating lesions have been found in the spinal cord $(3,4)$, basal ganglia $(5)$, midbrain, cerebral peduncle (6), internal capsule (7), and thalamus (8). Tonic spasms can be caused by lesions anywhere along the corticospinal pathways (9). Although tonic spasm is the most frequent movement disorder occuring in MS (10), it has not been a definitely described clinical entity for Devic's syndrome.

Devic's syndrome is an inflammatory demyelinating central nervous system disease with involvement of the optic nerves and spinal cord. However, inflammation and damage to the myelin can occur anywhere in the brain. Devic's syndrome may follow a monophasic or relapsing course. The most frequent initial manifestation is acute transverse myelitis. Acute transverse myelitis is caused by inflammation of the spinal cord and severe neurological dysfunction worsening over several hours to days. Furthermore, L'hermitte's symptom, radicular pain and paroxysmal tonic spasms can develop, especially in patients with relapsing NMO.

Levetiracetam is a new antiepileptic drug used as adjunctive treatment for partial onset with or without secondary generalization in epilepsy. Even though the underlying mechanism of action of levetiracetam has not been completely elucidated, the metabolism of GABA appears to be involved. Some reports indicate its effectiveness in the treatment of tardive dyskinesia, paroxysmal kinesigenic choreoathetosis and hemifacial spasm (11-14). To the best of our knowledge, this is an excellent drug for the treatment of patients with epilepsy and concomitant liver diseases (15).

We describe levetiracetam treatment for tonic spasms of a patient with Devic's Syndrome. The patient responded rapidly to the treatment.

\section{Case Report}

A 52-year old woman was admitted to our hospital with complaints of left leg pain, weakness, and dystonic posture. She said she had had right visual loss three months previously. She had a two weeks history of fever $\left(38.5^{\circ} \mathrm{C}\right)$. Two days later she developed tingling and numbness of her four limbs, followed by gait unsteadiness and urinary incontinence. Neurological examination showed an inability to walk unaided, due to truncal and limb ataxia and impaired vibratory sensation. All deep tendon reflexes were absent. The cranial nerves were normal. Fundus examination showed optic disk pallor, retro- 

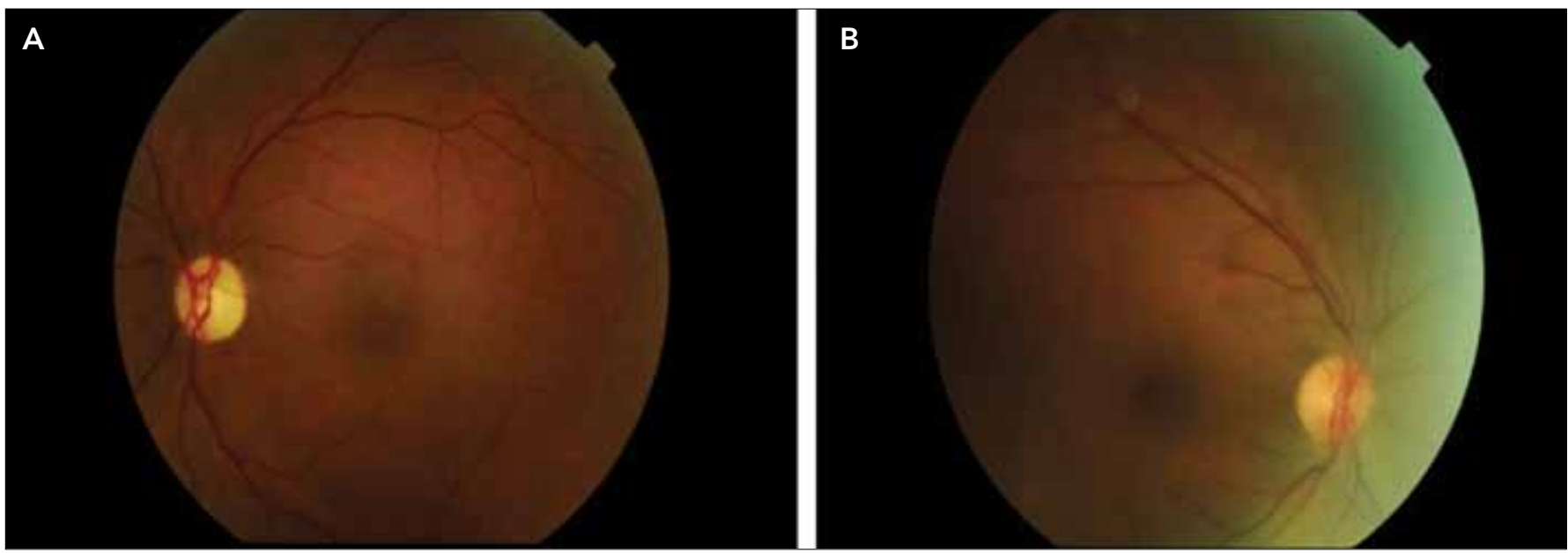

Figure 1. A) Fundus Picture of the Left Eye Shows Optic Disk Atrophy, B) Optic Disk Pallor in the Right Eye

bulbar neuritis in the right eye and optic disk atrophy in the left eye (Fig. 1 a and b). The CSF, examined 9 days after onset, was acellular with normal protein content. CSF IgG Index was within the normal range, whereas oligoclonal band was negative. MRI of the cranium was normal and cervical MRI showed an increased signal in the C5-T2 spinal cord (Fig. 2, 3). CSF protein was $50 \mathrm{mg} \%$ and glucose was $45 \mathrm{mg} \%$. In the serum, the anti CMV lgG titre was positive. Somatosensory evoked potentials showed poorly formed P40 cortical responses on stimulation of the left posterior tibial nerve. Left visual evoked potentials and bilateral posterior tibial SEP were absent. Right visual and brain-stem auditory evoked potentials were within normal limits. The electroencephalogram was normal; neither epileptiform activity nor changes in background were seen during or after tonic attacks. Based on the neurological sypmtoms, such as temporary blindness, quadriparesis and radiological findings, such as normal cranial MRI and spinal cord lesions extending over three or more vertebral segments, the patient was diagnosed to have Devic's syndrome. She was treated for 7 days with pulse dexamethasone.

The patient was reviewed by physiotherapists and the Rehabilitation Service where she had been hospitalized for three months because of continuing problems with weakness of both lower and upper limbs was noted. Under the treatment of intravenous seftriaxone for urinary infection, her condition was stable and she recovered gradually. Because tonic spasm was noted during her last admission, oral sodium valproate (Depakine, $1000 \mathrm{mg} /$ day) treatment was started. Her clinical course was uneventful, but seven days after taking Depakine, she had high fever and rash develop. Icteric sclera and yellowish discoloration of her skin were also noted thereafter. Correlating the clinical and laboratory findings, sodium valproate-induced hypersensitivity syndrome and hepatitis were diagnosed. Valproic acid treatment was stopped. Because of tonic spasm with pain, low dose carbamazepine therapy was started. After this treatment she developed cardiac arrest and was resuscitated. The patient was hospitalized in the intensive care unit for one month. The patient was transferred from the rehabilitation hospital to our neurology clinic because of pain and tonic spasm. Under this situation, the patient was started with oral Levatiracetam with low dosage (250 mg BID). After 2 weeks of therapy, the patient showed symptom regression of spasm with no adverse drug reactions.

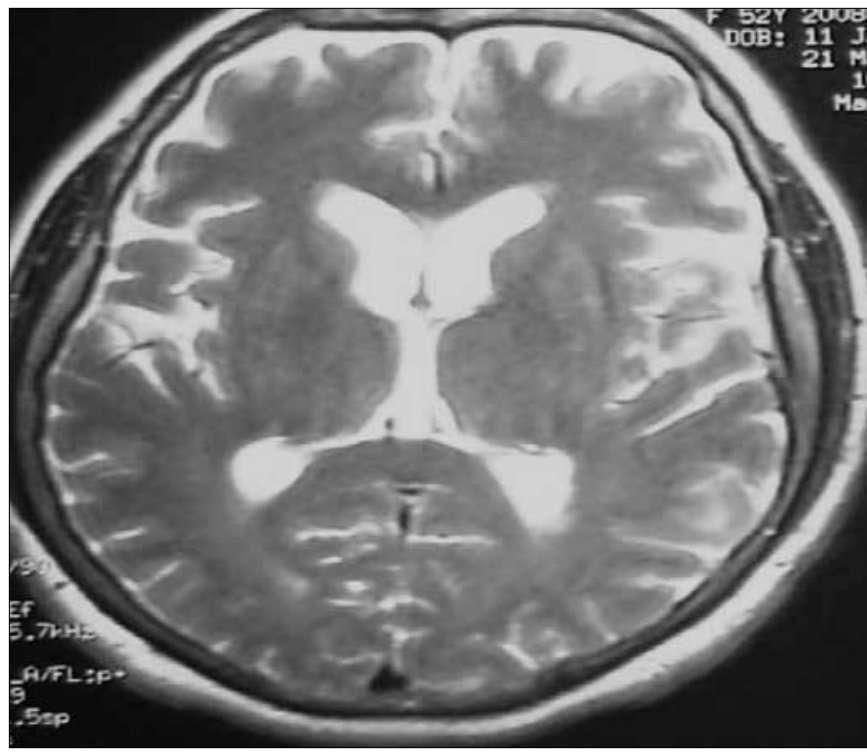

Figure 2. T2 Axial Cranial MR Image Shows No Abnormality

Generally, painful tonic spasms were induced by upper extremity hyperextension and followed by the ipsilateral extremite and body involvement. Spastic posture lasted a few minutes and spontaneous recovery of posture was seen. Tonic spasms responded partially to Depakine treatment but the drug was stopped because of the hypersensitivity reaction to the drug. Although Carbamazepine treatment controlled the spasms, after the development of cardiac arrest, the patient refused the drug. The last choice was Levatiracetam and it was used for 6 months. Tonic spasms respond dramatically to treatment with Levatiracetam. However there was a second attack six months later with quadriparesis. Plasmaphoresis and immunsupressive treatment were started and resulted in partial regression of tonic spasms. The patient is still receiving immunsupressive treatment.

\section{Discussion}

Tonic spasms are often apparent as paroxysmal attacks of unilateral painful tonic cramps of one or more limbs and last 


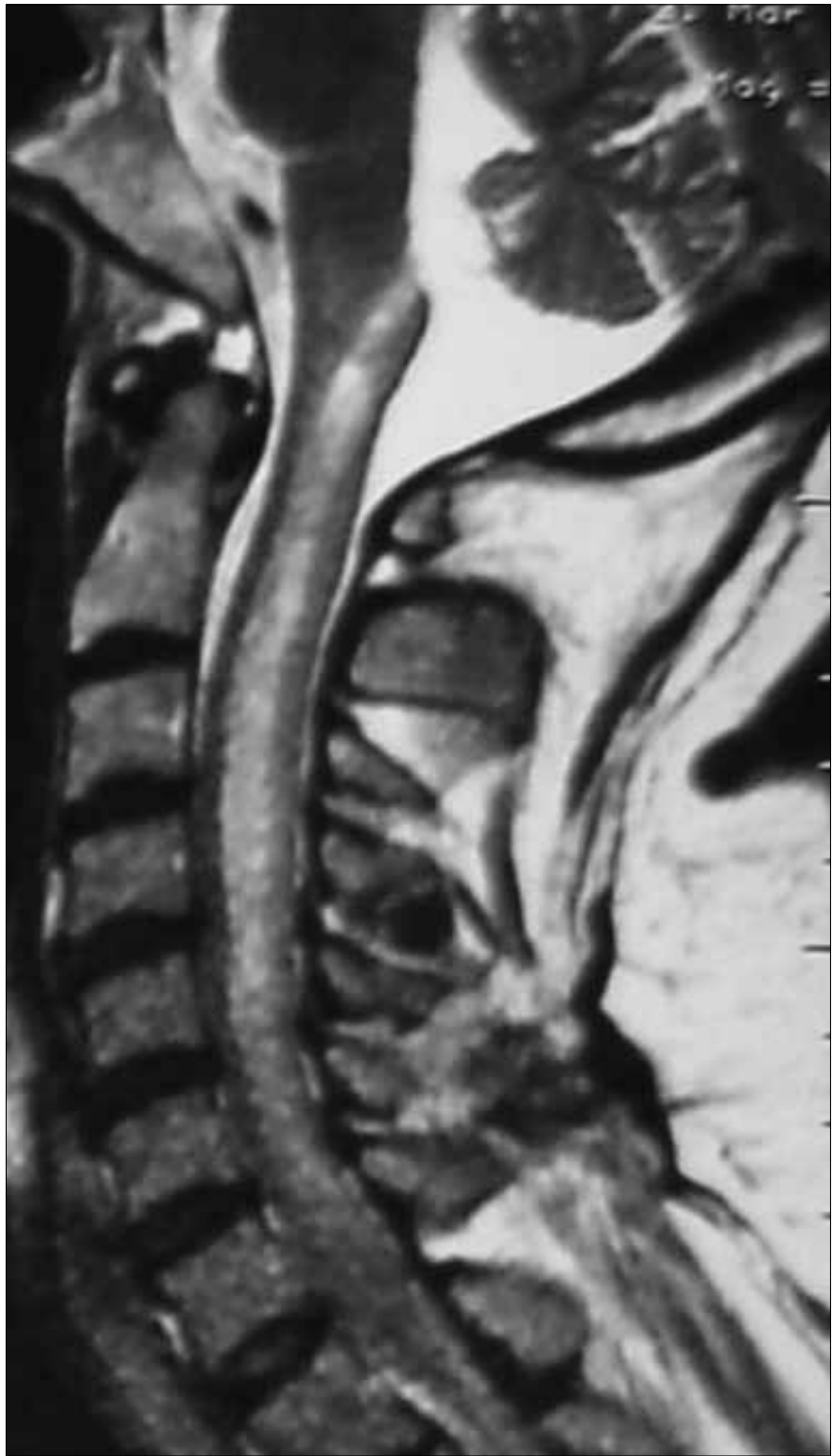

Figure 3. T2 Sagital Servical MR Image Shows Longitudinally Extensive Myelitis

for a few minutes. The clinical differential diagnosis for stereotyped tonic spasm includes epileptic seizures, psychogenic attacks, carpopedal spasm (tetany), paroxysmal kinesogenic choreoathetosis, and paroxysmal dystonic choreoathetosis, and the tonic spasms of demyelinating disease. Because of normal initial and ictal EEG, in our case clinical signs and corresponding EEG findings were characteristic for tonic spasm.

Paroxysmal non-epileptic symptoms seem to respond to many different drugs, but only a few well controlled studies have been conducted. Twomey and Espir published a study that suggests the use of carbamazepine in the treatment of paroxysmal symptoms of multiple sclerosis (16). Other antiepileptic drugs, such as valproic acid and phenytoin have also shown success (17). Recently an open-label trial of gabapentin for the treatment of paroxysmal symptoms in 18 patients with MS has been published (18). Successful use of bromocriptine and ibuprofen has also been reported (19-20).
Levetiracetam is a new antiepileptic drug approved for generalized and partial seizures. Hawker et al. reported that levetiracetam was effective in reducing phasic spasticity in their patients with MS (21). In our case, the patient with NMO had an excellent response to Levetiracetam therapy.

The aim of this article is to report the efficacy of levetiracetam controlling tonic spasms in neuromyelithis optica. Levetiracetam can be a new choice for treatment of painful tonic spasm with Devic's syndrome. Further studies are necessary to investigate the efficacy of levetiracetam on tonic spasms.

\section{Conflict of Interest}

No conflict of interest was declared by the authors.

\section{References}

1. Matthews WB. Symptoms and signs. In: Matthews WB et al. McAlpine's multiple sclerosis. Churchill Livingstone, Edinburgh, 1991;61-3.

2. Josef Spatt, Robert Chaix, Bruno Mamoli. Epileptic and non-epileptic seizures in multiple sclerosis. J Neurol 2001;248:2-9. [CrossRef]

3. Gatto EM, Zurru MC, Rugilo C. Medullary lesion and unusual bilateral paroxysmal dystonia in multiple sclerosis. Neurology 1996;46:847-8

4. Shibasaki H,Kuroiwa Y. Painful tonic seizures in multiple sclerosis Arch Neurol 1974;30:47-51.

5. Lugaresi A, Uncini A, Gambi D. Basal ganglia involvement in multiple sclerosis with alternating side paroxysmal dystonia. J Neurol 1993;240:257-8. [CrossRef]

6. Verheul GAM, Tyssen CC. Multiple sclerosis occurring with paroxysmal dystonia. Mov Disord 1990;5:352-5.

7. Maimone D, Reder AT, Finocchiaro F, Recupero E. Internal capsule plaque and tonic spasm in multiple sclerosis. Arch Neurol 1991;48:427-9.

8. Burghera JA, Català J, Casanova B. Thalamic demyelination and paroxysmal dystonia in multiple sclerosis. Mov Disord 1991;6:379-81.

9. Rose MR, Ball JA, Thompson PD. MR imaging in tonic spasm of MS. J Neurol 1993;241:115-7. [CrossRef]

10. Waubant E, Alize P, Tourbah A, Agid Y. Paroxysmal dystonia (tonic spasm) in multiple sclerosis Neurology 2001;57:2320-1.

11. Konitsiotis S, Pappa S, Mantas C, Mavreas V. Levetiracetam in tardive dyskinesia: an open label study. Mov Disord. 2006;21:1219-21. [CrossRef]

12. Deleu D. Levetiracetam in the treatment of idiopathic hemifacial spasm. Neurology. 2004;62:2134-5.

13. Costa J, Espírito-Santo C, Borges A, Ferreira JJ, Coelho M, Moore $\mathrm{P}$ et al. Botulinum toxin type A for hemifacial spasm. Cochrane Database Syst Rev 2005;25:CD004899

14. Biagio Carrieri P, Petracca M, Montella S. Efficacy of levetiracetam in hemifacial spasm: a case report. Clin Neuropharmacol. 2008;31:187-8. [CrossRef]

15. Glass GA, Stankiewicz J, Mithoefer A, Freeman R, Bergethon PR. Levetiracetam for seizures after liver transplantation. Neurology. 2005;64:1084-5.

16. Twomey JA, Espir MLE. Paroxysmal symptoms as the first manifestations of multiple sclerosis. J Neurol Neurosurg Psychiatry 1980;43:296-304. [CrossRef]

17. Espir MLE, Millac P. Treatment of paroxysmal disorders in multiple sclerosis with carbamazepin. J Neurol Neurosurg Psychiatry 1970;33:528-31.50411. [CrossRef]

18. Solaro C, Lunardi GL, Capello E, Inglese M, Messmer Uccelli M et al. An open-label trial of gabapentin treatment of paroxysmal symptoms in multiple sclerosis patients. Neurology 1998;51:609-11.

19. Kahn OA. Treatment of paroxysmal symptoms in multiple sclerosis with bromocriptine. J Neurol Neurosurg Psychiatry 1995;58:253.

20. Kahn OA. Treatment of paroxysmal symptoms in multiple sclerosis with ibuprofen. Neurology 1994;44:571-2.

21. Hawker K, Frohman E, Racke M. Levetiracetam for Phasic Spasticity in Multiple Sclerosis. Arch Neurol. 2003;60:1772-4. [CrossRef] 